\title{
Article
}

\section{Ultrathin Two-Dimensional Covalent Organic Framework Nanosheets: Preparation and Application in Highly Sensitive and Selective DNA Detection}

Yongwu Peng, Ying Huang, Yihan Zhu, Bo Chen, Liying Wang, Zhuangchai Lai, Zhicheng Zhang, Meiting Zhao, Chaoliang Tan, Nailiang Yang, Fangwei Shao, Yu Han, and Hua Zhang

J. Am. Chem. Soc., Just Accepted Manuscript • Publication Date (Web): 03 Jun 2017

Downloaded from http://pubs.acs.org on June 8, 2017

\section{Just Accepted}

"Just Accepted" manuscripts have been peer-reviewed and accepted for publication. They are posted online prior to technical editing, formatting for publication and author proofing. The American Chemical Society provides "Just Accepted" as a free service to the research community to expedite the dissemination of scientific material as soon as possible after acceptance. "Just Accepted" manuscripts appear in full in PDF format accompanied by an HTML abstract. "Just Accepted" manuscripts have been fully peer reviewed, but should not be considered the official version of record. They are accessible to all readers and citable by the Digital Object Identifier (DOI®). "Just Accepted" is an optional service offered to authors. Therefore, the "Just Accepted" Web site may not include all articles that will be published in the journal. After a manuscript is technically edited and formatted, it will be removed from the "Just Accepted" Web site and published as an ASAP article. Note that technical editing may introduce minor changes to the manuscript text and/or graphics which could affect content, and all legal disclaimers and ethical guidelines that apply to the journal pertain. ACS cannot be held responsible for errors or consequences arising from the use of information contained in these "Just Accepted" manuscripts. 


\title{
Ultrathin Two-Dimensional Covalent Organic Framework Nanosheets: Preparation and Application in Highly Sensitive and Selective DNA Detection
}

Yongwu Peng, ${ }^{\dagger} \S$ Ying Huang, ${ }^{\dagger} \S$ Yihan Zhu, ${ }^{\perp}$, Bo Chen $^{\dagger}$ Liying Wang, ${ }^{\star}$ Zhuangchai Lai, ${ }^{\dagger}$ Zhicheng Zhang, ${ }^{\dagger}$ Meiting Zhao, ${ }^{\dagger}$ Chaoliang Tan,${ }^{\dagger}$ Nailiang Yang, ${ }^{\dagger}$ Fangwei Shao, ${ }^{\dagger}$ Yu Han $,{ }^{*},{ }^{\perp}$ and Hua Zhang ${ }^{*},{ }^{\dagger}$

${ }^{\dagger}$ Center for Programmable Materials, School of Materials Science and Engineering, Nanyang Technological University, 50 Nanyang Avenue, Singapore 639798, Singapore

${ }^{\ddagger}$ Division of Chemistry and Biological Chemistry, School of Physical and Mathematical Sciences, Nanyang Technological University, Singapore 637371, Singapore

${ }^{\perp}$ Advanced Membranes and Porous Materials Center, Physical Sciences and Engineering Division, King Abdullah University of Science and Technology, Thuwal 23955-6900, Saudi Arabia

\author{
*Corresponding authors. E-mail: hzhang@ntu.edu.sg; yu.han@,kaust.edu.sa

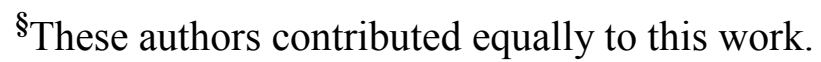

\begin{abstract}
The ability to prepare ultrathin two-dimensional (2D) covalent organic framework (COF) nanosheets (NSs) in high yield is of great importance for the further exploration of their unique properties and potential applications. Herein, by elaborately designing and choosing two flexible molecules with $C_{3 \mathrm{v}}$ molecular symmetry as building units, a novel imine-linked COF, namely TPA-COF, with hexagonal layered structure and sheet-like morphology, is synthesized. Since the flexible building units are integrated into the COF skeletons, the interlayer stacking becomes weak, resulting in the easy exfoliation of TPA-COF into ultrathin 2D NSs. Impressively, for the first time, the detailed structural information, i.e. the pore channels and individual building units in the NSs, is clearly visualized by using the recently developed low-dose imaging technique of transmission electron microscopy (TEM). As a proof-of-concept application, the obtained ultrathin COF NSs are used as a novel fluorescence sensing platform for the highly sensitive and selective detection of DNA.
\end{abstract}

\section{Introduction}

Covalent organic frameworks (COFs) are a class of porous crystalline materials with highly ordered two-dimensional (2D) or three-dimensional (3D) networks. ${ }^{1,2}$ COFs are built from the organic building 
blocks linked by reversible covalent bonds under reticular chemistry. ${ }^{3}$ Owing to their intriguing aesthetic architectures and unique properties, such as structural adaptivity and predictability, good hydrothermal stability, large surface area and extremely low density, COFs have shown great promising applications in gas storage ${ }^{4,5}$ and separation, ${ }^{6,7}$ optoelectronics, ${ }^{8-10}$ catalysis, ${ }^{11-16}$ etc. Recently, 2D COF nanosheets (NSs) have emerged as a new member in the family of $2 \mathrm{D}$ nanomaterials ${ }^{17-25}$ and received increasing attention with potential applications in chemical sensing, ${ }^{18}$ antimicrobial coatings, ${ }^{19}$ and cathode materials. $^{20}$

Normally, 2D COF NSs are prepared using the top-down strategy, in which COF NSs are obtained directly from their bulk counterparts via the solvent-assisted exfoliation, ${ }^{17,18,20,22}$ self-exfoliation, ${ }^{19}$ mechanical delamination ${ }^{21}$ or sequential post-synthetic modification. ${ }^{23}$ Although the aforementioned strategies have achieved great success in the preparation of COF NSs, the yield of obtained COF NSs is still low probably due to the strong interlayer $\pi-\pi$ stacking interactions. Recently, as reported by Banerjee et al. who used the cycloaddition reaction within the COF backbone, the interlayer $\pi-\pi$ stacking was destroyed, benefiting the exfoliation of bulk COF material to obtain 2D COF $\mathrm{NSs}^{24}$ Therefore, by directly integrating flexible building units into the backbone of COFs to weaken the interlayer stacking in COFs, the 2D COF NSs can be easily prepared after exfoliation.

Herein, by elaborately designing and choosing the geometries of building units and their connection patterns, a novel [3+3] imine-linked COF, namely TPA-COF, was successfully synthesized, which is constructed from two flexible building units with $C_{3 \mathrm{v}}$ molecular symmetry (Scheme 1, see Experimental Section for details). The TPA-COF possesses a hexagonal layered structure and sheet-like morphology. Impressively, The TPA-COF can be easily exfoliated into NSs, in which the ultrathin 2D nature allows direct imaging of the porous structure by our recently developed low-dose TEM technique. ${ }^{26}$ Importantly, the as-prepared 2D COF NSs can be used as a novel fluorescence sensing platform for highly selective and sensitive detection of DNA.

\section{Experimental Section}

Materials. 1,3,5-tris(4-formylphenyl)benzene (TFPB) was synthesized according to the published method. $^{22}$ Tris(4-aminophenyl)amine (TAPA, 98\%), tris(4-formylphenyl)amine (TFPA, 96\%), 1,2dichlorobenzene (o-DCB, 99\%), and acetic acid (AcOH, 99.5\%) were purchased from Tokyo Chemical Industry Co., Ltd. Ethanol (EtOH), acetone, dichloromethane and methanol with analytical purity were purchased from Merck. All DNA strands were synthesized and purified by the Integrated DNA Technologies Pte Ltd. The sequences of used DNA were listed in Table 1. Milli-Q water was obtained 
from the Milli-Q System (Millipore). All the materials were used as received without further purification.

\section{Synthesis of bulk TPA-COF material}

A $10 \mathrm{~mL}$ Pyrex tube was charged with tris(4-aminophenyl)amine (TAPA) (5 mg, $0.017 \mathrm{mmol})$, tris(4formylphenyl)amine (TFPA) $(5 \mathrm{mg}, 0.015 \mathrm{mmol})$ and $o$-DCB/ethanol $(4: 1 \mathrm{v} / \mathrm{v}, 1.5 \mathrm{~mL})$. After the mixture was sonicated for $5 \mathrm{~min}$, a clear solution was obtained. Subsequently, $0.06 \mathrm{~mL}$ of acetic acid (3 M) were added. Afterwards, the tube was flash frozen at $77 \mathrm{~K}$ using a liquid $\mathrm{N}_{2}$ bath and degassed by three freeze-pump-thaw cycles, sealed under vacuum and then heated at $120{ }^{\circ} \mathrm{C}$ for 3 days. A yellow precipitate was formed, which was collected by centrifugation and washed with anhydrous ethanol, anhydrous acetone, and anhydrous dichloromethane, separately. The collected sample was then solventexchanged with anhydrous methanol for 2-3 times and dried at $120^{\circ} \mathrm{C}$ under vacuum for $12 \mathrm{~h}$ to give a deep yellow powder $\left(6.5 \mathrm{mg}, 76 \%\right.$ isolated yield). IR (powder, $\left.\mathrm{cm}^{-1}\right)$ : 1694(w), 1619(m), 1594(s), 1505(s), 1427(w), 1318(m), 1284(m), 1197(w), 1169(m).

\section{Preparation of TPA-COF nanosheets (NSs)}

In a typical experiment, $6.5 \mathrm{mg}$ of bulk TPA-COF material were dispersed in $150 \mathrm{~mL}$ of ethanol. The mixture was sonicated in an ultrasonic bath (Brandson, CPX2800H-E, $110 \mathrm{~W}, 40 \mathrm{KHz}$ ) for $3 \mathrm{~h}$. After sedimentation for $24 \mathrm{~h}$, the upper colloidal suspension of exfoliated TPA-COF NSs was collected and concentrated by centrifugation at 7,000 rmp for $5 \mathrm{~min}$. After removal of supernatant, the residual TPACOF NSs were re-dispersed into $20 \mathrm{~mL}$ of ethanol prior to characterization.

\section{Gel electrophoresis analysis}

Hybridization chain reaction (HCR)-generated products were analyzed by the $1 \%$ agarose gel. The gel was run on the Bio-Rad horizontal electrophoresis system (Wide Mini-Sub Cell GT Cell) in 0.5× Trisborate-EDTA (TBE) buffer (45 mM Tris, $45 \mathrm{mM}$ boric acid and $1 \mathrm{mM}$ EDTA; pH 8.3) at $100 \mathrm{~V}$ for 90 min and stained with GelRed for $30 \mathrm{~min}$. Gel imaging was performed on a G:BOX system (Syngene) under UV irradiation.

\section{Fluorescent DNA assays}

In a typical hybrid experiment, $2.5 \mu \mathrm{L}$ of hairpin DNA probe $1(\mathrm{H} 1,10 \mu \mathrm{M})$ and $2.5 \mu \mathrm{L}$ of hairpin DNA probe $2(\mathrm{H} 2,10 \mu \mathrm{M})$ were incubated with $5 \mu \mathrm{L}$ of target DNA $(\mathrm{T}, 0-0.5 \mu \mathrm{M})$ in $440 \mu \mathrm{L}$ of sodium phosphate-sodium chloride buffer solution (SPSC buffer: $50 \mathrm{mM} \mathrm{Na} 2 \mathrm{HPO}_{4}$ and $0.75 \mathrm{M} \mathrm{NaCl}$; $\mathrm{pH} 7.4$ ) for $4 \mathrm{~h}$. Then $50 \mu \mathrm{L}$ of TPA-COF NS solution $\left(0.12 \mathrm{mg} \mathrm{mL}^{-1}\right)$ were added into the aforementioned mixture. After incubation for $15 \mathrm{~min}$, fluorescence measurements were performed to monitor the hybridization process with the final concentration of $\mathrm{T}(0-5 \mathrm{nM})$. The excitation and emission wavelengths were 590 and $609 \mathrm{~nm}$, respectively. 
For the comparison study, $2.5 \mu \mathrm{L}$ of hairpin DNA probe $1(\mathrm{H} 1,10 \mu \mathrm{M})$ and $2.5 \mu \mathrm{L}$ of hairpin DNA probe $2(\mathrm{H} 2,10 \mu \mathrm{M})$ were incubated with $5 \mu \mathrm{L}$ of target DNA $(\mathrm{T}, 0.5 \mu \mathrm{M})$ in $440 \mu \mathrm{L}$ of sodium phosphate-sodium chloride buffer solution (SPSC buffer: $50 \mathrm{mM} \mathrm{Na}_{2} \mathrm{HPO}_{4}$ and $0.75 \mathrm{M} \mathrm{NaCl}$; $\mathrm{pH}$ 7.4) for $4 \mathrm{~h}$. Then $50 \mu \mathrm{L}$ of bulk TPA-COF $\left(0.12 \mathrm{mg} \mathrm{mL}^{-1}\right)$ or TPA-COF NSs $\left(0.12 \mathrm{mg} \mathrm{mL}^{-1}\right)$ solution were added into the aforementioned mixture. After incubation for $15 \mathrm{~min}$, fluorescence measurements were performed to monitor the hybridization process with the final concentration of $\mathrm{T}(5 \mathrm{nM})$. The excitation and emission wavelengths were 590 and $609 \mathrm{~nm}$, respectively.

To study the selectivity of TPA-COF NS-based sensors, $2.5 \mu \mathrm{L}$ of hairpin DNA probe $1(\mathrm{H} 1,10 \mu \mathrm{M})$ and $2.5 \mu \mathrm{L}$ of hairpin DNA probe $2(\mathrm{H} 2,10 \mu \mathrm{M})$ were incubated with $5 \mu \mathrm{L}$ of target DNA $(\mathrm{T}, 0.5 \mu \mathrm{M}), 5$ $\mu \mathrm{L}$ of single-base mismatch DNA (SM, $0.5 \mu \mathrm{M})$, or $5 \mu \mathrm{L}$ of random DNA (R, $0.5 \mu \mathrm{M})$, respectively, in $440 \mu \mathrm{L}$ of sodium phosphate-sodium chloride buffer solution (SPSC buffer: $50 \mathrm{mM} \mathrm{Na}_{2} \mathrm{HPO}_{4}$ and 0.75 $\mathrm{M} \mathrm{NaCl}$; pH 7.4) for $4 \mathrm{~h}$. Then $50 \mu \mathrm{L}$ of TPA-COF NSs solution $\left(0.12 \mathrm{mg} \mathrm{mL}^{-1}\right)$ were added into the aforementioned mixtures. After incubation for $15 \mathrm{~min}$, fluorescence measurements were performed to monitor the hybridization process with the final concentration of T (5 nM), SM (5 nM) and R (5 nM), respectively. The excitation and emission wavelengths were 590 and $609 \mathrm{~nm}$, respectively.

\section{Characterization}

Prior to the TEM and AFM characterizations, the ethanolic suspension of TPA-COF NSs was dropped onto the holey carbon-coated carbon support copper grids and piranha-cleaned $\mathrm{Si} / \mathrm{SiO}_{2}$, respectively, and then naturally dried in air. For the XRD characterization, the TPA-COF NSs were concentrated and dropped on a clean glass. TEM images were obtained using a transmission electron microscope (JEOL JEM-2100F). AFM images were recorded using a dimension 3100 AFM with Nanoscope IIIa controller (Veeco, Fremont, CA) in tapping mode in air. The powder X-ray diffraction (PXRD) patterns of bulk COF materials were recorded on a Bruker D8 diffractometer (German) equipped with a $\mathrm{Cu} \mathrm{K} \alpha$ radiation $\left(\lambda=1.5406 \AA\right.$ ) at a scan rate of $0.02 \mathrm{deg} \mathrm{s}{ }^{-1}$. A Shimadzu XRD-6000 was used to characterize the XRD of TPA-COF NSs. Scanning electron microscopy (SEM) was observed on a JSM-7600F. Samples were treated with Pt sputtering before observation. Fourier transform infrared spectroscopy (FT-IR) spectra were collected on a Perkin Elmer FT-IR Spectrum GX in the spectral range of 400-4000 $\mathrm{cm}^{-1}$ using the $\mathrm{KBr}$ disk method. UV-Vis diffuse reflectance spectroscopy (UV-Vis DRS) spectra were obtained by a UV-Vis spectrophotometer (UV-Vis-NIR Cary 5000) and the data were converted to Kubelka-Munk functions for the band gap extraction. Thermogravimetric analyses (TGA) were performed using a TA Instrument TGA 2950 in the temperature range of 30 to $800{ }^{\circ} \mathrm{C}$ under flowing $\mathrm{N}_{2}\left(30 \mathrm{~mL} \mathrm{~min}^{-1}\right)$ with heating rate of $10{ }^{\circ} \mathrm{C} \mathrm{min}^{-1}$. The Brunauer-Emmett-Teller (BET) surface areas were calculated from $\mathrm{N}_{2}$ sorption isotherms at $77 \mathrm{~K}$ using a Micromeritics ASAP 2020 surface area and pore size analyser. Pore 
size distribution data were calculated based on the nonlocal density functional theory (NLDFT) model in the Micromeritics ASAP2020 software package.

\section{Low-dose TEM image acquisition, processing and simulation}

Low-dose HRTEM images were taken on a Cs-corrected FEI G ${ }^{2}$ cubed Titan 60-300 electron microscope under $300 \mathrm{kV}$, which is equipped with a Gatan K2 Summit direct-detection electroncounting camera (DDECC). The HRTEM images were similarly processed by using CRISP software in our previous work. $^{26}$ HRTEM image simulation was carried out by using multislice method implemented in QSTEM software (http://www.qstem.org). An accelerating voltage of $300 \mathrm{kV}$, a defocus value of $-300 \mathrm{~nm}$, a convergence angle of $0.2 \mathrm{mrad}$, a Cs value of $10 \mu \mathrm{m}$, and a focal spread of $5 \mathrm{~nm}$ were used. An information limit cutoff of $4 \AA$ was applied by using an objective aperture.

\section{Results and Discussion}

The tris(4-aminophenyl)amine (TAPA) and tris(4-formylphenyl)amine (TFPA) with $C_{3 \mathrm{v}}$ molecular symmetry are chosen as building units, since they can be further covalently connected through the $[3+3]$ imine condensation reaction. By condensing TAPA and TFPA in the mixture of $o$-dichlorobenzene $(o$ DCB)/ethanol/acetic acid (20:5:1, by vol.) at $120{ }^{\circ} \mathrm{C}$ for $72 \mathrm{~h}$ (Scheme 1), the bulk TPA-COF material was isolated as a deep yellow powder in approximately $76 \%$ yield which is insoluble in common organic solvents. The successful formation of imine linkages in bulk TPA-COF material was confirmed by the Fourier transform infrared spectroscopy (FT-IR, Figure S1), which exhibited a typical stretching band arising from $\mathrm{C}=\mathrm{N}$ at $1619 \mathrm{~cm}^{-1}$. Moreover, the aldehyde band of TFPA $\left(1694 \mathrm{~cm}^{-1}\right)$ was obviously attenuated, indicating the formation of imine-linked bonds. The UV-Vis diffuse reflectance spectroscopy (DRS) analysis revealed that the bulk TPA-COF material displays a reflection edge at around $530 \mathrm{~nm}$, corresponding to a band gap of $2.32 \mathrm{eV}$ (Figure S2). In addition, the thermogravimetric analysis (TGA) confirmed that the bulk TPA-COF material has high thermal stability up to $500{ }^{\circ} \mathrm{C}$ (Figure S3).

The crystalline structure and unit cell parameters of bulk TPA-COF material were determined by the powder X-ray diffraction (PXRD) analysis together with the structural simulation and Pawley refinement (Figure 1a and Table S1, S2 in Supporting Information). Considering the geometry of precursors and their connection patterns, two typical structural models with eclipsed and staggered 2D stacking and $P 3$ symmetry were built using the Materials Studio software package. ${ }^{27}$ The experimental PXRD profile of the bulk TPA-COF material (curve 1 in Figure 1a) matches well with the simulated pattern obtained using the eclipsed 2D stacking model both in the peak position and intensity (curve 4 in Figure 1a and Figure 1b), but is inconsistent with the PXRD pattern produced using staggered 2D stacking mode (curve 5 in Figure 1a). The bulk TPA-COF material exhibited an intense peak at 5.01 ${ }^{\circ}$ 
and minor peaks at $8.72^{\circ}, 10.13^{\circ}$ and $13.47^{\circ}$, which can be assigned to the diffractions of (100), (110), (200), and (210) planes, respectively. The presence of the reflection of the (001) plane in the bulk TPACOF material at $21.40^{\circ}$ indicates that the periodicity of $2 \mathrm{D}$ sheets is extended to the third dimension (c axis). To better understand the layer morphology of the bulk TPA-COF material and seek a closer correlation with the experimental PXRD pattern, the Pawley refinement was subsequently performed using the Materials Studio Forcite module. ${ }^{27}$ The Pawley-refined PXRD pattern (curve 2 in Figure 1a) is in good agreement with the experimental PXRD profile (curve 1 in Figure 1a), evident by their negligible difference as shown in the curve 3 in Figure 1a, with $R_{w p}$ and $R_{p}$ values converged to $1.64 \%$ and $4.68 \%$, respectively. The Pawley refinement yielded a trigonal unit cell of the bulk TPA-COF material with parameters of $\mathrm{a}=\mathrm{b}=20.9239 \AA, \mathrm{c}=3.92 \AA, \alpha=\beta=90^{\circ}$ and $\gamma=120^{\circ}$, which are close to the calculated lattice parameters $\left(\mathrm{a}=\mathrm{b}=21.1036 \AA, \mathrm{c}=3.92 \AA, \alpha=\beta=90^{\circ}\right.$ and $\left.\gamma=120^{\circ}\right)$ based on the predicted crystal structure. Such grid stack along the $\mathrm{c}$ axis leads to one dimensional open channels of $\sim 18 \AA$ and the interlayer separation of $3.92 \AA$ (Figure 1b). In the experimental PXRD profile (curve 1 in Figure 1a), the interlayer distance calculated from the (001) diffraction was estimated to be $4.1 \AA$, matching well with the simulated model (Figure 1b). Scanning electron microscopy (SEM) image confirmed the bulk TPA-COF material with sheet-like morphology (Figure 1c). The permanent porosity of the bulk TPA-COF material was determined by the $\mathrm{N}_{2}$ sorption isotherms measured at $77 \mathrm{~K}$ (Figure S4). It exhibited reversible type-I isotherms, with a pore volume of $0.89 \mathrm{~cm}^{3} \mathrm{~g}^{-1}$ and a BrunauerEmmett-Teller (BET) surface area of $1136.5 \mathrm{~m}^{2} \mathrm{~g}^{-1}$ (or $1489.4 \mathrm{~m}^{2} \mathrm{~g}^{-1}$ based on Langmuir model). By using the nonlocal density functional theory method, the pore size of the bulk TPA-COF material was determined to be $\sim 15 \AA$, which is close to the predicted pore size $(\sim 18 \AA)$ according to the crystal structure (Figure $1 \mathrm{~b}){ }^{18}$

Due to the flexible nature and sheet-like layered morphology of the bulk TPA-COF material, it was easily exfoliated into ultrathin 2D NSs, denoted as TPA-COF NSs, through one-step solvent-assisted liquid sonication (see Experimental Section for details). As shown in Figure 2a and Figure S5, SEM and TEM images clearly showed the 2D NS structures. A typical Tyndall effect was observed when a green laser went through the solution of TPA-COF NSs (inset in Figure 2a), confirming their colloidal structure. AFM characterization revealed that the thickness of the obtained TPA-COF NSs is $3.5 \pm 0.3 \mathrm{~nm}$ (Figure $2 \mathrm{~b}$ and Figure S6), corresponding to $9 \pm 1$ layers. To determine the crystal structure of TPA-COF NSs, the XRD characterization was performed. The result is identical to that of the bulk TPA-COF material, but the intensity of the first peak (100) decreased (Figure S7), which is consistent with the previous observation in the thin layered COFs. ${ }^{18,21,22,28}$ The FT-IR spectrum of TPA-COF NSs remains 
the same as the bulk material (Figure S8), further confirming the same structures of the bulk TPA-COF material and 2D TPA-COF NSs.

The ultrathin 2D nature of TPA-COF NSs allows us to visualize its honeycomb-like porous structure using high-resolution TEM (HRTEM). To the best of our knowledge, HRTEM image of COFs has rarely been reported until now, and there was only limited resolution achieved, mainly due to the easy structural damage of COFs under the electron beam. However, by using our recently developed lowdose TEM technique based on the use of a direct-detection electron-counting camera $^{26}$ (see Experimental Section for the detailed imaging conditions), a HRTEM image of TPA-COF NSs has been successfully acquired along the [001] direction with a total electron dose as low as $\sim 20 \mathrm{e}^{-} \mathrm{A}^{-2}$, containing useful structural information transferred up to $4 \AA$, as indicated by the fast Fourier transform (FFT, inset in Figure 2c). The hexagonally arranged white contrasts surrounded by six black dots are observed in the raw HRTEM image (Figure S9). The more clear denoised image is shown in Figure 2d, which matches well with the simulated HRTEM image (inset in Figure 2d, see Experimental Section for the simulation conditions) based on the proposed structure (Figure 1b). According to the simulation result, the observed white contrasts and black dots correspond to the 1D pore channels and the building units (TAPA and TFPA), respectively. Furthermore, we corrected the image based on the contrast transfer function (CTF) of the objective lens to make it more interpretable. In the CTF-corrected image (Figure 2e), each black dot in the raw image appears to be elongated along three directions into "three-claw" contrast, which becomes clearly identifiable by lattice averaging and symmetry imposing (Figure 2f, left). The processed image shows a perfect match with the projected potential map simulated from the proposed structure (Figure 2f, right), indicating that the "three-claw" corresponds to the three benzene rings of tridentate TAPA or TFPA molecules. To the best of our knowledge, this is the first time to directly observe the individual building units of a COF framework by HRTEM.

The successful exfoliation of bulk TPA-COF material to 2D TPA-COF NSs by solvent-assisted liquid sonication arises from the weak interlayer stacking interactions in bulk TPA-COF material, due to the flexible nonplanar conformation of building units. However, if TFPA in TPA-COF (Scheme 1) was replaced with 1,3,5-tris(4-formylphenyl)benzene (TFPB) during the reaction, another 2D layered COF, denoted as TPA-COF-2, was synthesized (see Experimental Section in Supporting Information for details), whose structure was confirmed by PXRD (Figure S10a and Table S3, S4 in Supporting Information) and FT-IR (Figure S11). Compared to the bulk TPA-COF material, the bulk TPA-COF-2 material exhibits a similar crystal structure (Figure S10b) and morphology (Figure S12), but is difficult to be exfoliated into NSs (Figure S13), which may come from the increasing interlayer $\pi$ - $\pi$ stacking 
interactions in TFP-COF-2 compared to TFP-COF, due to the nearly planar conformation and strong $\pi$ systems of TFPB.

As known, 2D NSs, such as graphene, ${ }^{29-33}$ graphitic carbon nitride $\left(\mathrm{g}-\mathrm{C}_{3} \mathrm{~N}_{4}\right),{ }^{34}$ transition metal dichalcogenides (TMDs) ${ }^{35-38}$ and metal-organic frameworks (MOFs), ${ }^{39}$ have shown promising applications in fluorescence sensors. As a proof-of-concept application, the ultrathin TPA-COF NS was used as a novel sensing platform for DNA detection. The proposed strategy is shown in Figure 3a. Two hairpin DNA probes, namely $\mathrm{H} 1$ and $\mathrm{H} 2$, were designed, and $\mathrm{H} 1$ was labeled with a fluorescent dye. H1 and $\mathrm{H} 2$ can be adsorbed on the surface of TPA-COF NSs due to the $\pi$ - $\pi$ stacking interactions, resulting in the fluorescence quenching of dye. However, when the target DNA (T) is present, it can specifically trigger a hybridization chain reaction (HCR) between $\mathrm{H} 1$ and $\mathrm{H} 2$ as described previously, ${ }^{40}$ yielding the long double-stranded DNA (dsDNA), which has very weak interaction with TPA-COF NSs. Therefore, the HCR-generated long dsDNA leaves away from the surface of TPA-COF NSs, resulting in the recovery of fluorescence of dye, which can provide the quantitative detection of target DNA.

The DNA sequences used here are listed in Table 1. As shown in Figure 3b, the solution containing H1 and H2 exhibited strong emission at the wavelength of $609 \mathrm{~nm}$ (curve I). After addition of TPA-COF NSs into the solution, the fluorescence was quenched, and 91\% quenching efficiency were obtained in 15 min (curve IV and blue curve of inset in Figure 3b), indicating the strong fluorescence quenching ability of TPA-COF NSs. In contrast, when T triggered the HCR between $\mathrm{H} 1$ and H2 to form the long dsDNA, the fluorescence was greatly retained even in the presence of TPA-COF NSs (curve II and green curve of inset in Figure 3b). However, the fluorescence was slightly retained when only H1 was used as hairpin probe due to one-step hybridization between H1 and T (curve III), suggesting that the recovery of fluorescence signal indeed resulted from the target DNA-triggered HCR. In addition, the formation of HCR-generated long dsDNA was confirmed by the gel electrophoresis (Figure S14). Compared to the bulk TPA-COF material (Figure S15), the TPA-COF NSs exhibited better fluorescence quenching ability (Figure 3c). The fluorescence quenching ability was enhanced from the TPA-COF bulk material to the TPA-COF NSs, which could be attributed to the increased surface area of TPA-COF NSs. $^{18}$

As shown in Figure 3d and Figure S16a, the fluorescence intensity enhanced with the concentration of $\mathrm{T}$, and exhibited a linear relationship in the range of $0-1 \mathrm{nM}$ with the detection limit of $20 \mathrm{pM}$, which is comparable with or even better than most of 2D nanomaterial-based fluorescence DNA sensors (Table 2). Importantly, the selectivity of proposed DNA sensor was also evaluated by using the single-based mismatch DNA (denoted as SM) and random DNA (denoted as R). As shown in Figure S16b in 
Supporting Information, T caused the distinct increase of fluorescence signal compared to the SM and $\mathrm{R}$, indicating the excellent selectivity of our TPA-COF NS-based fluorescence sensor.

\section{Conclusions}

In summary, we report the synthesis and characterization of a novel [3+3] imine-linked layered COF, namely TPA-COF, by elaborately designing and choosing two flexible molecules with $C_{3 \mathrm{v}}$ molecular symmetry. Impressively, the obtained bulk TPA-COF material, with a highly ordered hexagonal network and sheet-like morphology can be easily exfoliated into ultrathin nanosheets (NSs) possibly due to the weak interlayer stacking originating from the flexible nonplanar building units. Importantly, the ordered pore channels and building units on the pore walls are simultaneously observed in the TPA-COF NSs by our recently developed low-dose TEM technique. As a proof-of-concept application, the ultrathin 2D TPA-COF NS was used as a novel fluorescence sensing platform for the detection of DNA with excellent selectivity and high sensitivity. Our unique strategy can be used to design, synthesize and characterize other ultrathin 2D COF NSs, which might have various promising applications in membrane separation, drug delivery and electronic devices.

\section{ASSOCIATED CONTENT}

Supporting Information

Additional experimental procedures, figures, tables and crystallographic data (PDF and CIF). The Supporting Information is available free of charge on the ACS Publications website at http://pubs.acs.org.

\section{AUTHOR INFORMATION}

Corresponding Author

*hzhang@ntu.edu.sg

*yu.han@kaust.edu.sa

\section{Author Contributions}

${ }^{\S}$ These authors contributed equally to this work.

\section{Notes}

The authors declare no competing financial interest.

\section{ACKNOWLEDGMENT}


This work was supported by MOE under AcRF Tier 2 (ARC 19/15, No. MOE2014-T2-2-093; MOE2015-T2-2-057; MOE2016-T2-2-103) and AcRF Tier 1 (2016-T1-001-147; 2016-T1-002-051), NTU under Start-Up Grant (M4081296.070.500000) and iFood Research Grant (M4081458.070.500000), and Singapore Millennium Foundation in Singapore. We would like to acknowledge the Facility for Analysis, Characterization, Testing and Simulation, Nanyang Technological University, Singapore, for use of their electron microscopy facilities.

\section{REFERENCES}

(1) Côté, A. P.; Benin, A. I.; Ockwig, N. W.; O'Keeffe, M.; Matzger, A. J.; Yaghi, O. M. Science 2005, 310, 1166-1170.

(2) El-Kaderi, H. M.; Hunt, J. R.; Mendoza-Cortés, J. L.; Côté, A. P.; Taylor, R. E.; O'Keeffe, M.; Yaghi, O. M. Science 2007, 316, 268-272.

(3) Huang, N.; Wang, P.; Jiang, D. Nat. Rev. Mater. 2016, 1, 16068.

(4) Doonan, C. J.; Tranchemontagne, D. J.; Glover, T. G.; Hunt, J. R.; Yaghi, O. M. Nat. Chem. 2010, 2, 235-238.

(5) Zeng, Y.; Zou, R.; Zhao, Y. Adv. Mater. 2016, 28, 2855-2873.

(6) Kandambeth, S.; Biswal, B. P.; Chaudhari, H. D.; Rout, K. C.; Kunjattu, H. S.; Mitra, S.; Karak, S.; Das, A.; Mukherjee, R.; Kharul, U. K.; Banerjee, R. Adv. Mater. 2017, 29, 1603945-1603954.

(7) Fu, J.; Das, S.; Xing, G.; Ben, T.; Valtchev, V.; Qiu, S. J. Am. Chem. Soc. 2016, 138, 7673-7680.

(8) Dogru, M.; Bein, T. Chem. Commun. 2014, 50, 5531-5546.

(9) Huang, N.; Ding, X.; Kim, J.; Ihee, H.; Jiang, D. Angew. Chem. Int. Ed. 2015, 54, 8704-8707.

(10) Vazquez-Molina, D. A.; Mohammad-Pour, G. S.; Lee, C.; Logan, M. W.; Duan, X.; Harper, J. K.; Uribe-Romo, F. J. J. Am. Chem. Soc. 2016, 138, 9767-9770.

(11) Vyas, V. S.; Haase, F.; Stegbauer, L.; Savasci, G.; Podjaski, F.; Ochsenfeld, C.; Lotsch, B. V. Nat. Commun. 2015, 6, 8508.

(12) Li, H.; Pan, Q.; Ma, Y.; Guan, X.; Xue, M.; Fang, Q.; Yan, Y.; Valtchev, V.; Qiu, S. J. Am. Chem. Soc. 2016, 138, 14783-14788.

(13) Xu, H.; Gao, J.; Jiang, D. Nat. Chem. 2015, 7, 905.

(14) Lin, S.; Diercks, C. S.; Zhang, Y.; Kornienko, N.; Nichols, E. M.; Zhao, Y.; Paris, A. R.; Kim, D.; Yang, P.; Yaghi, O. M.; Chang, C. J. Science 2015, 349, 1208-1213.

(15) Wang, X.; Han, X.; Zhang, J.; Wu, X.; Liu, Y.; Cui, Y. J. Am. Chem. Soc. 2016, 138, 12332-12335.

(16) Aiyappa, H. B.; Thote, J.; Shinde, D. B.; Banerjee, R.; Kurungot, S. Chem. Mater. 2016, 28, 43754379. 
(17) Berlanga, I.; Ruiz-González, M. L.; González-Calbet, J. M.; Fierro, J. L.; Mas-Ballesté, R.; Zamora, F. Small 2011, 7, 1207-1211.

(18) Das, G.; Biswal, B. P.; Kandambeth, S.; Venkatesh, V.; Kaur, G.; Addicoat, M.; Heine, T.; Verma, S.; Banerjee, R. Chem. Sci. 2015, 6, 3931-3939.

(19) Mitra, S.; Kandambeth, S.; Biswal, B. P.; Khayum, M. A.; Choudhury, C. K.; Mehta, M.; Kaur, G.; Banerjee, S.; Prabhune, A.; Verma, S.; Roy, S.; Kharul, U. K.; Banerjee, R. J. Am. Chem. Soc. 2016, $138,2823-2828$.

(20) Wang, S.; Wang, Q.; Shao, P.; Han, Y.; Gao, X.; Ma, L.; Yuan, S.; Ma, X.; Zhou, J.; Feng, X.; Wang, B. J. Am. Chem. Soc. 2017, 139, 4258-4261.

(21) Chandra, S.; Kandambeth, S.; Biswal, B. P.; Lukose, B.; Kunjir, S. M.; Chaudhary, M.; Babarao, R.; Heine, T.; Banerjee, R. J. Am. Chem. Soc. 2013, 135, 17853-17861.

(22) Bunck, D. N.; Dichtel, W. R. J. Am. Chem. Soc. 2013, 135, 14952-14955.

(23) Mitra, S.; Sasmal, H. S.; Kundu, T.; Kandambeth, S.; Illath, K.; Díaz, D. D.; Banerjee, R. J. Am. Chem. Soc. 2017, 139, 4513-4520.

(24) Khayum, M. A.; Kandambeth, S.; Mitra, S.; Nair, S. B.; Das, A.; Nagane, S. S.; Mukherjee, R.; Banerjee, R. Angew. Chem. Int. Ed. 2016, 55, 15604-15608.

(25) Dai, W.; Shao, F.; Szczerbiński, J.; McCaffrey, R.; Zenobi, R.; Jin, Y.; Schlüter, A. D.; Zhang, W. Angew. Chem. Int. Ed. 2016, 55, 213-217.

(26) Zhu, Y., Ciston, J., Zheng, B., Miao, X., Czarnik, C., Pan, Y., Sougrat, R., Lai, Z., Hsiung, C.E., Yao, K., Pinnau, I., Pan, M., Han, Y. Nat. Mater. 2017, 16, 532.

(27) Accelrys Materials Studio Release Notes, 5.5, Accelrys Software, Inc., San Diego, 2010.

(28) Biswal, B. P.; Chandra, S.; Kandambeth, S.; Lukose, B.; Heine, T.; Banerjee, R. J. Am. Chem. Soc. 2013, 135, 5328-5331.

(29) Lu, C.; Yang, H.; Zhu, C.; Chen, X.; Chen, G. Angew. Chem. Int. Ed. 2009, 48, 4785-4787.

(30) He, S.; Song, B.; Li, D.; Zhu, C.; Qi, W.; Wen, Y.; Wang, L.; Song, S.; Fang, H.; Fan, C. Adv. Funct. Mater. 2010, 20, 453-459.

(31) Liu, X.; Aizen, R.; Freeman, R.; Yehezkeli, O.; Willner, I. ACS Nano 2012, 6, 3553-3563.

(32) Liu, X.; Wang, F.; Aizen, R.; Yehezkeli, O.; Willner, I. J. Am. Chem. Soc. 2013, 135, 11832-11839.

(33) Qian, Z.; Shan, X.; Chai, L.; Ma, J.; Chen, J.; Feng, H. Nanoscale. 2014, 6, 5671-5674.

(34) Wang, Q.; Wang, W.; Lei, J.; Xu, N.; Gao, F.; Ju, H. Anal. Chem. 2013, 85, 12182-12188.

(35) Zhu, C.; Zeng, Z.; Li, H.; Li, F.; Fan, C.; Zhang, H. J. Am. Chem. Soc. 2013, 135, 5998-6001.

(36) Huang, J.; Ye, L.; Gao, X.; Li, H.; Xu, J.; Li, Z. J. Mater. Chem. B. 2015, 3, 2395-2401.

(37) Yuan, Y.; Li, R.; Liu, Z. Anal. Chem. 2014, 86, 3610-3615. 
(38) Zhang, Y.; Zheng, B.; Zhu, C.; Zhang, X.; Tan, C.; Li, H.; Chen, B.; Yang, J.; Chen, J.; Huang, Y.; Wang, L.; Zhang, H. Adv. Mater. 2015, 27, 935-939.

(39) Zhao, M.; Wang, Y.; Ma, Q.; Huang, Y.; Zhang, X.; Ping, J.; Zhang, Z.; Lu, Q.; Yu, Y.; Xu, H.; Zhao, Y.; Zhang, H. Adv. Mater. 2015, 27, 7372-7378.

(40) Dirks, R. M.; Pierce, N. A. Proc. Natl. Acad. Sci. 2004, 101, 15275-15278. 
Scheme 1. Schematic illustration of synthesis and extended hexagonal structure of the bulk TPACOF material

$2 \quad$ COF material

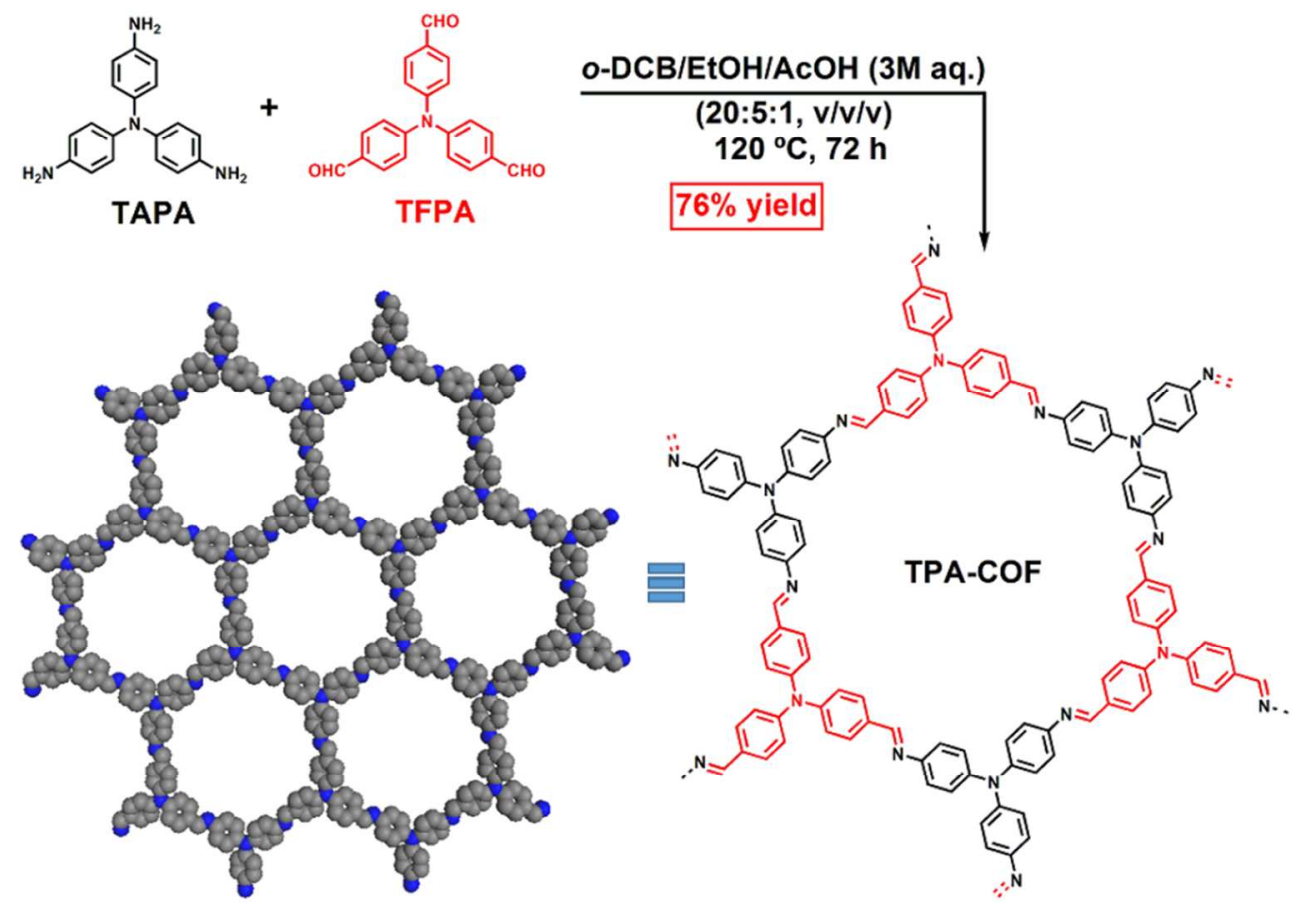


3) Difference of curves 1 and 2

4) TPA-COF_eclipsed, simulated

5) TPA-COF_staggered, simulated
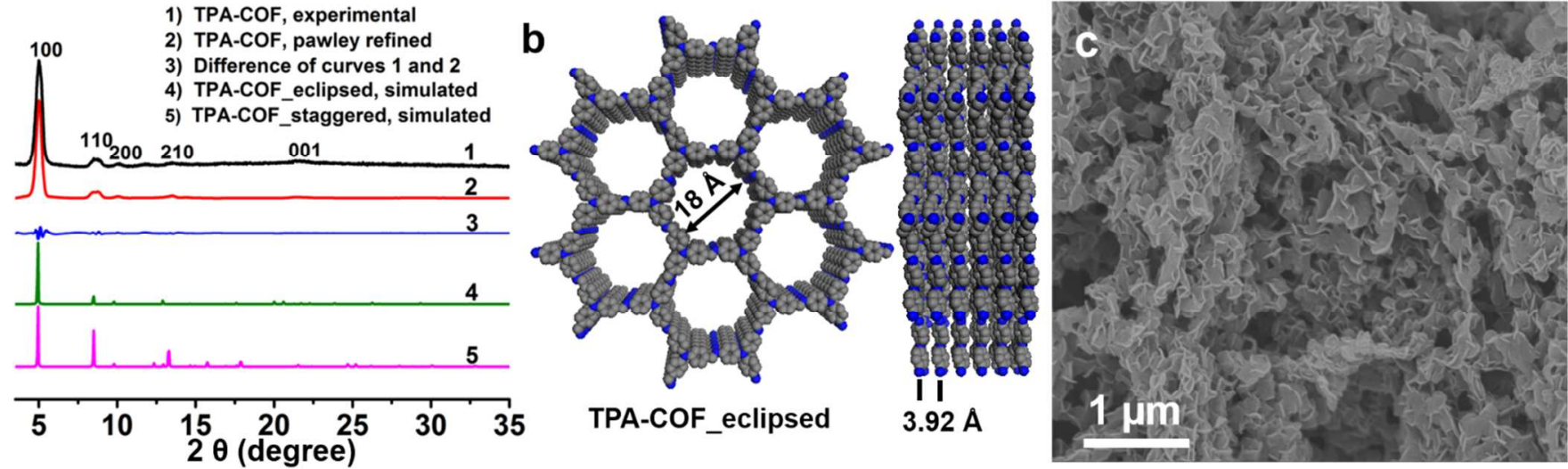

Figure 1. Characterization of layered TPA-COF bulk material. (a) Experimental and simulated PXRD patterns of the bulk TPA-COF material. (b) Crystal structure of the bulk TPA-COF material assuming the eclipsed stacking viewed along [001] (left) and [100] (right) directions with the interlayer distance of $3.92 \AA$. (c) SEM image of the bulk TPA-COF material with sheet-like morphology. 

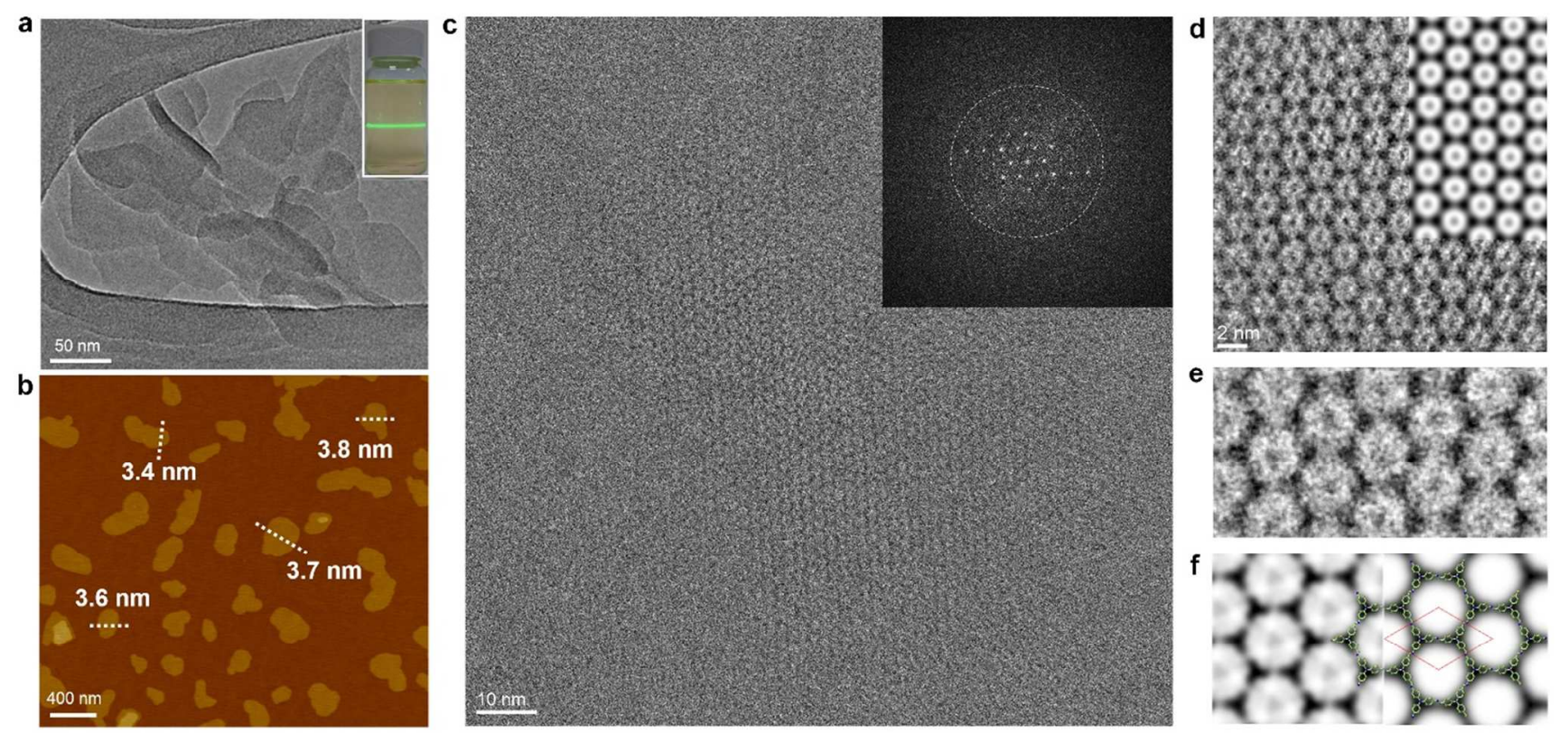

Figure 2. Characterization of 2D TPA-COF NSs. (a) TEM image of TPA-COF NSs. Inset: Photograph of Tyndall effect of the TPA-COF NS suspension. (b) AFM image of TPA-COF NSs with the thickness indicated. (c) Low-dose high-resolution motion-corrected TEM image of a typical TPA-COF NS. Inset: FFT from the blue dashed circle on the TPA-COF NS (indicated in Figure S9), in which a white dashed circle marks the information limit of $4 \AA$. (d) Enlarged HRTEM image denoised by using a Wiener filter. Insert: Simulated HRTEM image. (e) Contrast transfer function (CTF) corrected and denoised HRTEM image based on a -300 nm defocus value determined from an amorphous region. (f) Left: lattice-averaged, P3-symmetry imposed and CTF-corrected HRTEM image. Right: Simulated projected potential map with a point spread function width of $4 \AA$, in which the projected structural model in green and unit cell in red are embedded. 
a
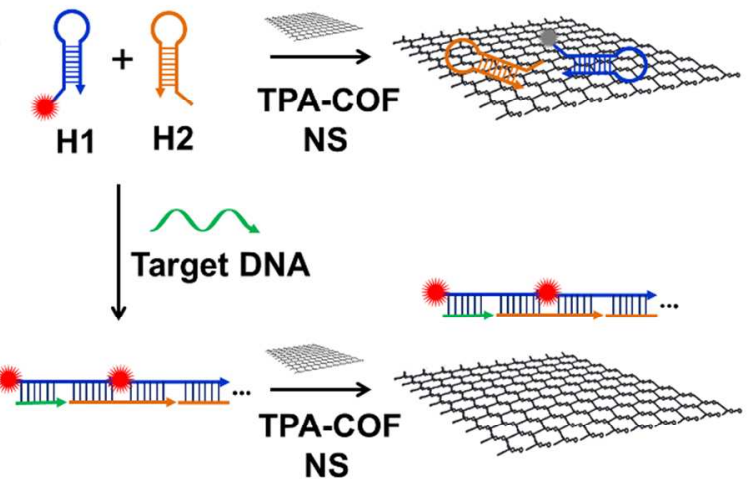

NS

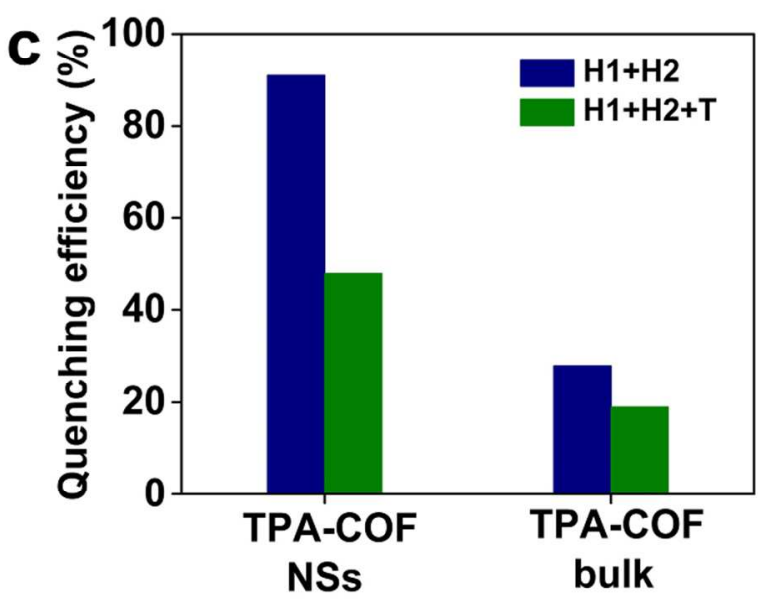

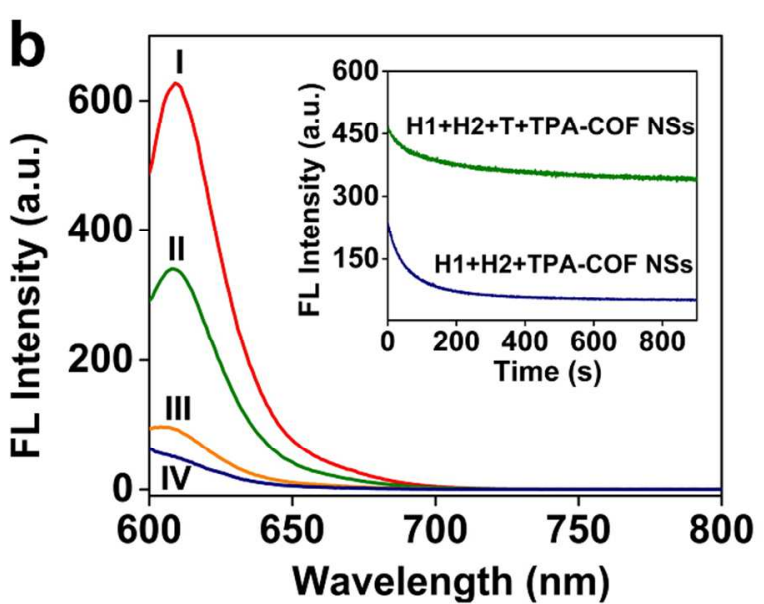

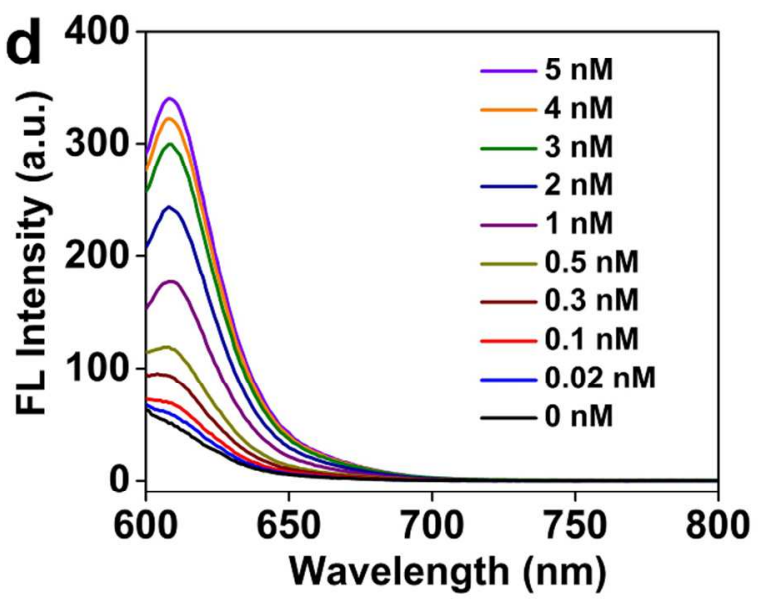

Figure 3. Detection of DNA with TPA-COF NSs. (a) Schematic illustration of TPA-COF NSbased fluorescence sensor for detection of DNA. (b) Fluorescence spectra under different experimental conditions: (I) $\mathrm{H} 1+\mathrm{H} 2$; (II) $\mathrm{H} 1+\mathrm{H} 2+\mathrm{T}+\mathrm{TPA}-\mathrm{COF} \mathrm{NSs}$; (III) H1+T+TPA-COF NSs; and (IV) H1+H2+TPA-COF NSs. The concentrations of H1, H2, T and TPA-COF NSs in the final solution are $50 \mathrm{nM}, 50 \mathrm{nM}, 5 \mathrm{nM}$, and $12 \mu \mathrm{g} \mathrm{mL}{ }^{-1}$, respectively. Inset: Kinetic study on the fluorescence change of $\mathrm{H} 1+\mathrm{H} 2$ and $\mathrm{H} 1+\mathrm{H} 2+\mathrm{T}$ in the presence of TPA-COF NSs. Excitation and emission wavelengths are 590 and $609 \mathrm{~nm}$, respectively. (c) The fluorescence quenching efficiency of TPA-COF NSs and bulk TPA-COF. The concentrations of H1, H2, T, TPA-COF NSs and bulk TPA-COF material in the final solution are $50 \mathrm{nM}, 50 \mathrm{nM}, 5 \mathrm{nM}, 12 \mu \mathrm{g} \mathrm{mL}^{-1}$, and $12 \mu \mathrm{g} \mathrm{mL} \mathrm{m}^{-1}$, respectively. (d) Fluorescence spectra of the proposed sensing platform in the presence of different concentrations. The concentrations of H1, H2, and TPA-COF NSs in the final solution are $50 \mathrm{nM}, 50 \mathrm{nM}$, and $12 \mu \mathrm{g} \mathrm{mL}^{-1}$, respectively. 
Table 1. DNA sequences used for the fluorescence assay

\begin{tabular}{ll}
\hline Name & Sequence (5'-3') \\
\hline Hairpin probe 1 (H1) & Texas Red-TTAACCCACGCCGAATCCTAGACTCAAAGTAGTCTAGGATTCGGCGTG \\
Hairpin probe 2 (H2) & AGTCTAGGATTCGGCGTGGGTTAACACGCCGAATCCTAGACTACTTTG \\
Target DNA (T) & AGTCTAGGATTCGGCGTGGGTTAA \\
Single-base mismatch & AGTCTAGGATTCAGCGTGGGTTAA \\
DNA (SM) & \\
Random DNA (R) & TATCGCAAGGCGCATACCGGGTCG \\
\hline
\end{tabular}

Table 2. Comparison of fluorescent DNA sensors using different 2D nanomaterials

\begin{tabular}{|c|c|c|c|c|}
\hline 2D nanomaterials & $\begin{array}{l}\text { Fluorescent } \\
\text { reporter }\end{array}$ & Sensitivity & Comments & Ref. \\
\hline Graphene oxide (GO) & FAM & $2 \mathrm{nM}$ & - & 29 \\
\hline GO & FAM, ROX, Cy5 & $100 \mathrm{pM}$ & Multiplexed analysis & 30 \\
\hline GO & FAM, ROX & $5 \mathrm{pM}$ & $\begin{array}{l}\text { Exonuclease III amplification and } \\
\text { multiplexed analysis }\end{array}$ & 31 \\
\hline GO & $\begin{array}{c}\text { Silver } \\
\text { nanoclusters }\end{array}$ & $0.5 \mathrm{nM}$ & Multiplexed analysis & 32 \\
\hline GO & $\begin{array}{l}\text { Graphene } \\
\text { quantum dots }\end{array}$ & $75 \mathrm{pM}$ & - & 33 \\
\hline $\mathrm{g}-\mathrm{C}_{3} \mathrm{~N}_{4}$ & FAM, ROX & $81 \mathrm{pM}$ & Exonuclease III amplification & 34 \\
\hline $\mathrm{MoS}_{2}$ & FAM & $0.5 \mathrm{nM}$ & - & 35 \\
\hline $\mathrm{MoS}_{2}$ & FAM & $15 \mathrm{pM}$ & Hybridization chain reaction & 36 \\
\hline $\mathrm{WS}_{2}$ & TAMRA & $60 \mathrm{pM}$ & - & 37 \\
\hline $\mathrm{TaS}_{2}$ & FAM, Texas red & $50 \mathrm{pM}$ & Multiplexed analysis & 38 \\
\hline Cu-TCPP MOF NSs & Texas red, TET & $20 \mathrm{pM}$ & Multiplexed ananlysis & 39 \\
\hline TPA-COF NSs & Texas red & $20 \mathrm{pM}$ & Hybridization chain reaction & $\begin{array}{l}\text { This } \\
\text { work }\end{array}$ \\
\hline
\end{tabular}

Abbreviations: FAM, carboxyfluorescein; ROX, 6-carboxy-x-rhodamine; Cy5, cyanine 5; TET, tetrafluororescein; TAMRA, tetramethylrhodamine. 


\section{Graphic Content}

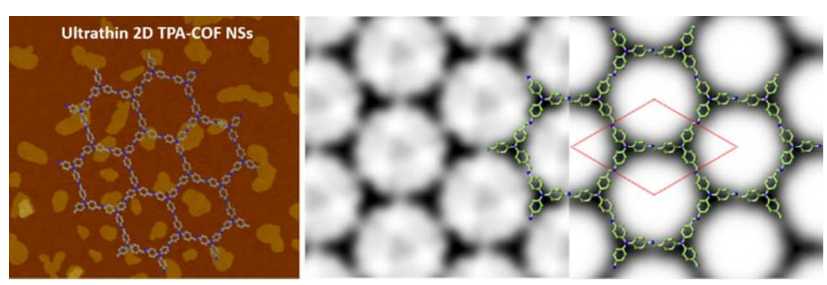




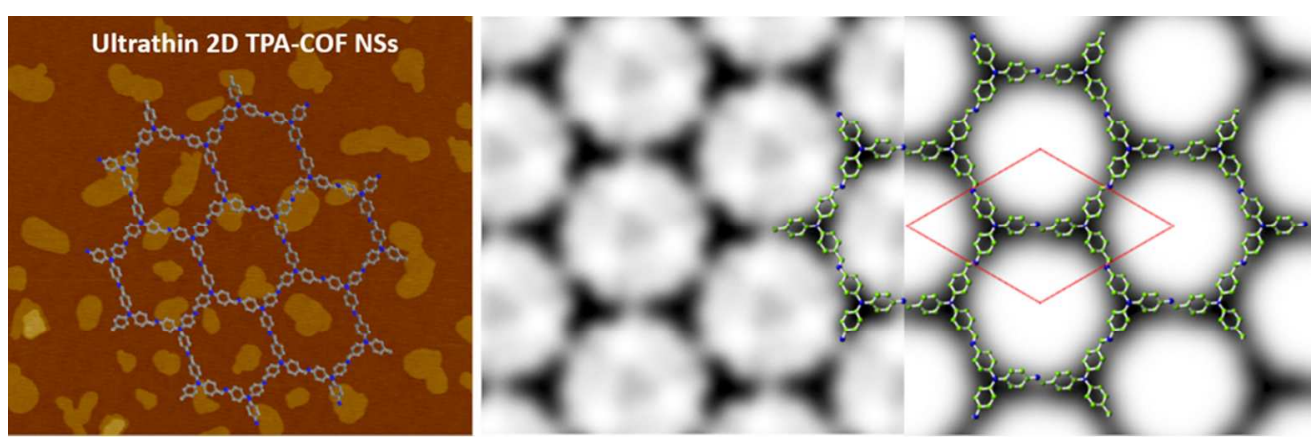

TOC image

$85 \times 27 \mathrm{~mm}(300 \times 300$ DPI $)$ 